\title{
Frequency of nosocomial infections with antibiotic resistant Acinetobacter spp. in intensive care unit (ICU) patients
}

\author{
Amini Maryam ${ }^{1 *}$, Jalali Nadooshan Mohammadreza ${ }^{2}$, Davati Ali $^{3}$ and Golestanifard Mahdieh ${ }^{4}$ \\ ${ }^{1}$ Department of Infectious Diseases and Tropical Medicine, Faculty of Medicine, Shahed University, Shaheed Mustafa \\ Khomeini Hospital, Tehran, Iran. \\ ${ }^{2}$ Department of Pathology, Faculty of Medicine, Shahed University, Teharn, Iran. \\ ${ }^{3}$ Department of Social Medicine, Faculty of Medicine, Shahed University, Teharn, Iran. \\ ${ }^{4}$ Faculty of Medicine, Shahed University, Teharn, Iran.
}

Accepted 23 April, 2012

\begin{abstract}
Acinetobacter is a Gram-negative coccobacillus and one of the most nosocomial infections. Acinetobacter spp resistant strains in the world have created health problems. The aim of this study was to determine the prevalence and associated risk factors for infections with multi drug resistance (MDR) of Acinetobacter spp. in patients admitted to the ICU of Shahid Mostafa Khomeini Hospital, Tehran, Iran, 2008 to 2010 . This descriptive-analytical and cross-sectional study was designed on all of the 130 patients with culture positive for Acinetobacter spp. Microbiologic and specific demographic data were extracted from patient's laboratory and archive file. The data were analyzed by using SPSS16 statistical software and chi-square and Mann-Whitney test. The prevalence of infection with Acinetobacter spp. separately by years was: $21.5,30.8$ and $47.7 \%$ in 1386,1387 and 1388 , respectively. $100 \%$ isolates were resistance to Carbnicillin, Piperacillin, Cefotaxime and Cephalotin, $99.2 \%$ to Ciprofloxacin, Cotrimoxazole and Chloramphenicol, 97.7\% to Imipenem, 95.4\% to Tetracycline and 91.5\% to Gentamicin. Highest percentage sensitivity was to Amikacin $14.6 \%$. Nosocomial infections with Acinetobacter spp. during the three years, was a growing trend and all isolates were MDR and the highest susceptibility was to Amikacin. It seems that the incorrect diagnosis, use of antibiotics for viral infections, inappropriate doses and time of antibiotic therapy (less or more), inappropriate formulation and low quality of some of antibiotics, are the most important cause of MDR. The proper use of antibiotics to prevent MDR bacterial nosocomial infections is recommended.
\end{abstract}

Key words: Acinetobacter spp, intensive care unit (ICU), multi drug resistance (MDR), nosocomial infections.

\section{INTRODUCTION}

Nosocomial infection refers to infection that developed during hospitalization and not during the incubation period at admission (Akbari et al., 2005). It is well known that hospital infection is a health problem of modern societies. The important causes of nosocomial infections are Acinetobacter spp. (Khosroshahi and Sharifi, 2007). Acinetobacter spp, are Gram-negative, aerobic, nonmandatory and encapsulated coccobacillus within family Moraxellaceae. Bacteria do not require special conditions

\footnotetext{
*Corresponding author. E-mail: mmamini55602@gmail.com.
}

to grow and grows in any $\mathrm{pH}$ and temperature. Transmission occurs from person to person (colonized or patient) or after contact with contaminated environment. Acinetobacter spp are the most common Gram-negative microorganisms are constantly on the levels and health care worker skin (Khosroshahi and Sharifi 2007; www.infectious disease, accessed 2008). Previous studies showed that risk factors for nosocomial Acinetobacter spp. infection include: Increased length of hospital stay, surgery, wounds, broad-spectrum antibiotic therapy, parenteral nutrition, intravascular or urinary catheter, hospitalization in the intensive care unit (ICU) or burn unit, intubation and mechanical ventilation and risk 
factors for community-acquired Acinetobacter spp. infection include: Alcoholism, smoking, renal failure, chronic lung disease and diabetes (Bennani et al., 2008). Acinetobacter spp. infections are detectable by blood, sputum, urine, wounds, and cerebrospinal fluid culture. Antimicrobial susceptibility can be investigated by various methods in which the method considered the gold is standard agar dillusion (www.infectious disease, accessed 2008). .But one major problem is, the ability of microorganisms by the variety mechanisms to gain resistance to antibiotics and the emergence of strains that are resistant to all commercially available antibiotics (Wareham et al., 2008). The main concern there is that beta-lactamase producing Acinetobacter spp. include serine and metallobeta-lactamase which are resistant to Carbapenems (Munoz-price and Weinstein 2008; Mshana et al., 2009). Carbapenem resistant Acinetobacter spp. can cause treatment problem because Carbapenems is the core of treatment for resistant Gram-negative infections (Zarrilli et al., 2004). According to Patwardhan et al. (2008) multi drug resistance strain of $A$. baumannii (MRAB) resistant to all beta-lactams, fluoroquinolones and aminoglycoside, although it usually is polymyxin-sensitive, but the pan resistant (resistant to all antibiotics except Colistin) has been reported by Patwardhan et al. (2008). The emergence of resistant strains of Acinetobacter spp. leading to increased length of hospital stay, mortality and healthcare costs (Jen et al., 2008; Sunenshine et al., 2007). The prevalence of infections with antibiotic resistant Acinetobacter spp. and their association with some factors such as length of hospital stay, recent hospital admission and surgery, history of previous antibiotic use has not been determined in ICU patients of Shahid Mostafa Khomeini Hospital in Tehran. So to describe the prevalence and risk factors of Acinetobacter spp. in more details we conducted the present study on all of ICU patients with culture positive sample for Acinetobacter spp. during a three-year period. To take away from understanding underlying factors, the overall prevalence, antibiotic resistance, length of hospital stay, overall mortality rates and hospital costs resulting from hospital infections to be reduced.

\section{MATERIALS AND METHODS}

This was a descriptive-analytical and cross-sectional study of 130 patients with Acinetobacter spp. culture positive sample and aged between 12 to 92 years admitted to ICU ward of Shahid Mostafa Khomeini Hospital, Tehran Iran from 2008 to 2010. All culture samples (including blood, urine, wound, sputum) grown by standard methods bacteriology were positive for patients with Acinetobacter spp. who have been admitted into the hospital for at least two to three days. The samples were selected and studied over a threeyear period (from 2008 to 2010). Disk diffusion method was used for antibiogram to determine antibiotic resistance of Acinetobacter spp.. Antibiotics has been used for antibiogram include: Amikacin, Gentamicin, Carbnicillin, Piperacillin, Imipenem, Cotrimoxazole, Tetracycline, Cefotaxime, Cephalotine, Ciprofloxacin and
Chloramphenicol.

Then, in patients with positive cultures of Acinetobacter spp. factors such as length of hospital stay, underlying disease, history of hospitalization, recent surgery and antibiotic therapy, was obtained. Based on the definitions of National Nosocomial Infection Surveillance (NNIS) those patients had no symptoms of infection or were cultures in the hospital less than two to three days of admission or was incomplete information on their records, and all patients with negative culture or culture positive with another bacteria were excluded. The data were analyzed by using SPSS16 statistical software and chi-square and Mann-Whitney test .We considered differences significant at $p<0.05$.

\section{RESULTS}

Of 130 patients with positive cultures of Acinetobacter spp. were 70 males (53.8\%) and 60 females (46.2\%). The average age was $17.19 \pm 68.8$ years with range between 92 and 12 years. The most infection rates was observed at ages above 50 years. The prevalence of infection with Acinetobacter spp. based on year was as follows: Of 130 samples, about 28 (21.5\%), 40 (30.8\%) and $62(47.7 \%)$ in 2008, 2009 and 2010, year, respectively. Of the total samples, $117(90 \%)$ samples were sputum, $9(6.9 \%)$ wound, 3(2.3\%) urine and blood $1(0.8 \%)$. Average length of stay in hospital is 31.7 days. Of the total patients studied, 91 patients $(70 \%)$ had a history of hospitalization during the past 6 months and 39 (30\%) had no history of previous hospitalizations.

103 patients $(79.2 \%)$ had underlying disease and 27 case $(20.8 \%)$ had no underlying disease. Similarly, 41 patients (31.5\%) had a history of surgery within the past 6 months and 89 case $(68.5 \%)$ had no such history.

In 95 patients (73.1\%), history of previous antibiotic therapy was seen and 35 patients $(26.9 \%)$ had no such history. Of 117 sputum samples, in 112 cases (95.7\%) patient were connected to the ventilator. Antibiotic resistance of Acinetobacter spp was high compared to most antibiotics that are given in Table 1.

Highest sensitivity was to Amikacin (14.6\%). Also, $100 \%$ of isolates were resistant to three classes of antibiotics is the indicator for multi drug resistance. The results showed that antibiotic resistance with an underlying disease, length of hospital stay, history of admission, recent surgery and antibiotic treatment before infection, had no meaningful relationship.

\section{DISCUSSION}

Our study demonstrated the prevalence of hospital infection with Acinetobacter spp. in three years on a growing trend, and all isolates had multiple drug resistance. The most probable explanation for this increasing trend is the incorrect use of antibiotics to treat viral infections, incorrect diseases identifying, incorrect doses of antibiotics, inappropriate treatment duration (less or more than been recommended time), arbitrary use of antibiotics, prescription of antibiotics by unaware 
Table 1. The frequency of resistance and sensitivity to various antibiotics against Acinetobacter spp.

\begin{tabular}{lcccc}
\hline Antibiotic & Sensitivity number & Resistancy number & Sensitivity (\%) & Resistancy (\%) \\
\hline Carbnicillin & 0 & 130 & 0 & 100 \\
Piperacillin & 0 & 130 & 0 & 100 \\
Cefotaxime & 0 & 130 & 0 & 100 \\
Cephalotine & 0 & 130 & 0 & 100 \\
Ciprofloxacin & 1 & 129 & 0.8 & 99.2 \\
Cotrimoxazole & 1 & 129 & 0.8 & 99.2 \\
Chloramphenicol & 1 & 129 & 0.8 & 99.2 \\
Imipenem & 3 & 127 & 2.3 & 97.7 \\
Tetracycline & 6 & 124 & 4.6 & 95.4 \\
Gentamicin & 11 & 119 & 8.5 & 91.5 \\
Amikacin & 19 & 111 & 14.6 & 85.4 \\
\hline
\end{tabular}

persons, inappropriate formulation and low quality of some of antibiotics. Results of our study indicate that respiratory infections were the most common source of clinical isolates of Acinetobacter spp. which has also been observed in the Qorban Ali and colleagues study's (Ghorbanalizadegan et al., 2007). But in studies by other researchers, after urinary tract infection, pneumonia was the second most common infection in hospitals (Flanders et al., 2006). Elderly people (aged above 50 years) due to a weak immune system were most at risk to infections and risk of nosocomial infections by opportunistic microorganisms. In ICU admitted patients due to severe illness, long-term hospitalization and use of invasive procedures risk for infection is high (Ghorbanalizadegan et al., 2007). In our study, average age $17.19 \pm 68.8$ years were infected with Acinetobacter spp. and most of these infections were age over 50 years. Based on the findings of this study, the prevalence of infection with Acinetobacter spp. increases from $21.5 \%$ in year 2008 to $47.7 \%$ in year 2010 . Today it is proven that the majority of Acinetobacter spp. resistance to beta lactam antibiotics, quinolones, and aminoglycosides resistance that is growing (Van Looveren and Goossens, 2004). According to the results of the study, Acinetobacter spp. was most resistance to Carbnicillin, piperacillin, cefotaxime, cephalotin and most low-level resistance to amikacin and gentamicin, respectively; our results support those of Sadeghifard et al. in years 2004 to 2006, in which was determined that all Acinetobacter spp. isolates, were resistant to cephalosporins, Carbnicillin and Ticarcillin and $44 \%$ of isolates were susceptible to amikacin (Sadeghifar et al., 2006). However the results are in disagreement with those of Basustaoglu et al. (2001) in which all isolates of the strains of Acinetobacter bomany showed susceptibility to Imipenem, which is in sharp contrast with the results of this study (Basustaogl et al., 2001). One of the important characteristics of Acinetobacter spp. strains resistant to multiple classes of antibiotics creates many problems in the treatment of hospital infections (Khaltabadi et al., 2009). In our study, all isolates had multiple drug resistance which is more than the results of the study done by Hujer et al. (2006) in which $89 \%$ of isolates were resistant to at least three classes of antibiotics which is the standard for multi drug resistance. But, in Sadeghifard et al. (2006) study, 100\% of isolates had multiple drug resistance, which is agreement with our results. Hospital infections are important because of increasing incidence, mortality, cost and economic losses (Ghorbanalizadegan et al., 2007). Hospital infection control, though costly, difficult and time consuming, it is necessary and affordable. The emergence of resistant strains of Acinetobacter spp. lead to increased length of hospitalization, medical expenses and mortality (Jen et al., 2008; Sunenshine et al., 2007). So recognition, inhibition and the introduction of various resistance mechanisms used by strains of hospital acquired Acinetobacter spp, would be of importance. We recommend further studies using a larger sample to investigate global prevalence and risk factors of Acinetobacter spp. infections.

\section{ACKNOWLEDGEMENTS}

The author expresses their thanks to Miss Mahdiye Golestanifard and Dr. Jalali Nadoushan, chairman of laboratory ward, and other staff of Shahid Mostafa Khomeini hospital for their kind attention and cooperation.

\section{REFERENCES}

Akbari M, Davoudzade M, Rozbahani H, Tarhi M, Bayat A, Radsari E (2005). Types of pathogens isolated from hospital staff gowns Martyrs Khorramabad tribes in 2003-2004 years. Lorestan Univ. Med. Sci. J. 7(2):11-16.

Acintobacter (2008). Available at: http://www.infectiousdisease. Accessed (2008). Louisiana.dhh.gov.

Bennani B, Selmani R, Mahmoud M, Nejjari C, Kanjaa N (2008). Nosocomial pneumonia in mechanically ventilated patients: prospective study in intensive care unit of Fez university hospital. Saudi J. Anaes. 2(2):46-51.

Basustaoglu AC, Kisa O, Sacilik SC (2001). Epidemiological 
characterization of hospital-acquired Acinetobacter baumannii isolates from a 1500-bed teaching hospital by phenotypic and genotypic methods. J. Hosp. Infec. 47:246-249.

Flanders S, Collard H, Saint S (2006). Nosocomial pneumonia: state of the science. Am. J. Infect. Control 34:84-93.

Ghorbanalizadegan M, Ranjbar R, Izadi M, Esmaili D, Ahmadi A, Goudarzi Z (2007). Prevalence of Pseudomonas aeruginosa and Acinetobacter with multi drug resistance in patients admitted to hospital Baghiatollah. Ilam Univ. Med. Sci. J. 15(1):1-5.

Hujer KM, Hujer AM, Hulten EA, Bajaksouzian S, Adams JM, Donskey CJ (2006). Analysis of antibiotic resistance genes in multi drug resistant Acintobacter spp. isolates from military and civilian patients treated at the walter reed army medical center. J. Antimicrob. Chemother. 50(12):4114-4123.

Jen SMU, Yao INA, Chun IHS, Ming $\mathrm{CCH}$, Jung $\mathrm{WCH}$, Ling $\mathrm{CPO}$ (2008). Risk factor of multi drug resistance in nosocomial bacteremia due to Acinetobacter baumannii. J. Microb. Immunol. Infect. 41:118123.

Khosroshahi N, Sharifi M (2007). Carbapenem resistant Acinetobacter strains isolated from patients in intensive care units and equipment of health centers in Qazvin in 2006-2007. Iran. J. Med. Microb. 3(1):3338.

Khaltabadi R, Moniri R, Shajari G, Nazem M, Mousavi G, Ghasemi A, (2009). The pattern of antibiotic resistance and spread of antibiotic resistance genes in Acinetobacter strains isolated from patients, Kashan. Faiz J. Med. Res. 12(4):60-66.

Munoz-price LS, Weinstein RO (2008). Acintobacter infection. N. Eng. J. Med. 358:1271-281.

Mshana ST, Kamugisha ER, Mirambo MA, Chakraborty TR, Lyamuya EL (2009). Prevalence of multi resistant gram negative organisms in a tertiary hospital in Mwanza, Tanzania. BMC J. Res. Notes 2:49-54
Patwardhan R, Dhakephalkar P, Niphadkar K, Chopade B (2008). A Study of nosocomial pathogen in ICU with special reference to multi resistant Acintobacter baumannii harbouring multiple plasmids. Ind. J. Med. Res. 128:178-187.

Sunenshine RH, Marc-Oliver W, Lisa LM, Anthony DH, Xiaoyan S, Joan H, Sara EC, Ashley A, Jennifer C, Daniel BJ, David GK, Trish MP, Harold CS, Arjun S (2007). Multi drug resistant Acintobacter infection mortality rate and length of hospitalization. J. Emer. Infect. Dis. 13(1):97-103.

Sadeghifard N, Ranjbar R, Ghasemi A, Pakzad I, Zaimi J, Zameri A (2006). Evaluation of drug resistant strains of Acinetobacter baumannii and other species isolated from three hospitals, Tehran. Ilam Univ. Med. Sci. J. 14(3):29-36.

Van LM, Goossens H (2004). Antimicrobial resistance of Acinetobacter spp in Europe. J. Clin. Microb. Infect. 10:684-704.

Wareham D, Bean D, Khanna P, Hennessy E, Krahe D, Ely A (2008). Blood stream infection due to Acintobacter spp: epidemiology, risk factor and impact of multi drug resistance. Eur. J. Clin. Microb. Infect. Dis. 27:607-612.

Zarrilli RA, Crispino MA, Bagattini MA, Barretta EL, Di Popolo AN, Triassi MA (2004). Molecular epidemiology of sequential out breaks of $A$. baumannii in ICU shows the emergence of carbapenem resistance. J. Clin. Microb. 42(3):946-953. 